\title{
Acetylation of ATP5f1c mediates cardiomyocyte senescence via metabolic dysfunction in radiation induced heart damage
}

\author{
Zhimin Zeng \\ The Second Affiliated Hospital of Nanchang University \\ Peng $\mathrm{Xu}$ \\ The Second Affiliated Hospital of Nanchang University \\ Yanqing $\mathrm{He}$ \\ The Second Affiliated Hospital of Nanchang University \\ Yali Yi \\ The Second Affiliated Hospital of Nanchang University

\section{Zhicheng Liu} \\ The first Clinical College of Nanchang University \\ Jing Cai \\ The Second Affiliated Hospital of Nanchang University \\ Long Huang \\ The Second Affiliated Hospital of Nanchang University \\ Anwen Liu ( $\sim$ awliu666@163.com ) \\ The Second Affiliated Hospital of Nanchang University
}

\section{Research Article}

Keywords: lonizing radiation, heart disease, Atp $5 f 1 c$, lysine acetylation, metabolic dysfunction, senescence

Posted Date: February 4th, 2022

DOI: https://doi.org/10.21203/rs.3.rs-1312353/v1

License: @) (7) This work is licensed under a Creative Commons Attribution 4.0 International License. Read Full License 


\section{Abstract}

Objective: Ionizing radiation (IR) causes heart senescence, which eventually manifests as radiation-induced heart damage (RIHD). Energy metabolism of cardiomyocytes is involved in senescence. This study attempts to explore radiation-induced senescence mechanisms through TMT-labeled proteomics.

Methods: Echocardiography, senescence-associated secretory phenotype (SASP), telomere length and P21 expression were conducted to detect IR induced senescence. Total protein acetylation of irradiated heart apex and $\mathrm{H} 9 \mathrm{C} 2$ cells was discovered by Western blotting compared with the control. To further investigate lysine acetylation alterations in irradiated heart proteins, proteomics of lysine acetylation was performed. Acetylated level of Atp5f1c was verified by Co-IP. The plasmids of the acetylated-mimicking Atp5f1c mutant (K55Q), deacetylated-mimicking Atp5f1c mutant (K55R) and WT were constructed and used for to transfect $\mathrm{H} 9 \mathrm{C} 2$ cells to prove that acetylated Atp5f1c participated in energy metabolism and senescence. Deacetylation inhibitors, plasmid transfection and Co-IP were used to determine the mechanism of upstream regulation of Atp5f1c K55-Ac.

Results: LVEF was decreased, SASP was elevated, telomere length was shortened and P21was overexpressed after IR. In addition, the total protein acetylation level significantly increased, and 69 proteins, including 90 sites, showed a lysine acetylation response to irradiation. The majority of the hyperacetylated proteins were related to energy metabolism. The ATP synthase y subunit was hyperacetylated at the 55th lysine site and led to decreased ATP enzyme activity and synthesis decreased. Atp5f1c K55-Ac induced H9C2 metabolic dysfunction and senescence. Sirt4 and Sitr5, deacetylase inhibitors, mediated Atp5f1c K55Ac deacetylation.

Conclusion: IR induced cardiomyocyte senescence and energy metabolism disorder. Atp5f1c K55-ac, which is modulated by Sirt4 or Sirt5, might be a potential molecular target underlying RIHD.

\section{Introduction}

Radiotherapy has become one of the most important treatments for multiple cancers. It was reported that over half of patients have received radical or palliative radiotherapy during the anticancer process[1]. Unfortunately, patients obtain therapeutic benefits from radiotherapy while ionizing radiation may bring normal organ complications to patients at the same time[2,3]. Radiation-induced heart damage (RIHD) is mainly observed after radiotherapy for malignant chest tumors, especially left breast cancer and Hodgkin's lymphoma with high definitive cure rates[4, 5]. RIHD is mainly characterized by coronary artery disease, ischemic heart disease, pericarditis, conduction defects, and valvular dysfunction and so on[6, 7]. The risk of RIHD was a significant concern in present oncology practice therapy.

Senescence plays a vital role in embryogenesis, host immunity, tumor suppression and other physiological and pathological processes[8]. Cardiac cellular senescence induced by inflammation or ROS mostly presents with telomere shortening, DNA damage, functional decline, and other molecular changes[9]. Cytokines, chemokines, growth factors, and proteases produced by inflammation or ROS injury are called senescence-associated secretory phenotypes (SASPs). Previous studies have demonstrated that the p53/p21 signaling axis is involved in the regulation of ionizing-induced senescence[10, 11].Dysregulation of energy metabolism is relevant to aging-related cardiac diseases[12, 13]. Ionizing radiation increased endothelial P21 expression [14],and total-body irradiated (TBI) mice received 9 Gy ionizing radiation manifested with cardiac and kidney senescence[15].Thus, ionizing radiation might cause cardiac senescence. However, the molecular mechanisms underlying radiation induced senescence remain unclear.

Lysine acetylation of proteins is a conserved posttranslational modification that modifies protein structures and integrates biological processes such as metabolism, circadian rhythm and gene transcription in organisms[16, 17]. Molecular mechanisms of acetylated protein alterations have been studied in many cardiac diseases [18]. The protein acetylation level is regulated by lysine acetyltransferases (KATs) and deacetylases (KDACs)[19]. Some KATs or HDACs regulate energy metabolism and have been intensively studied in mammals[16]. In addition, the acetylation level of proteins is especially relevant for some metabolic diseases including atherosclerosis, diabetes mellitus and polycystic ovary syndrome[20, 21]. Recently, Masaya Tsuda et al. proved that lysine acetylation is a vital mechanism underlying skeletal muscle metabolism[22]. Apoe ${ }^{-/-}$mice that received 6 Gy total body irradiation presented with an increase in cardiac mitochondrial protein acetylation level and metabolic damage, which were related to a decrease in Sirt1 [23]. However, the relationship between the acetylation level of proteins and radiation-induced cardiac senescence is still unclear.

In this study, we aimed to investigate the mechanisms of radiation-induced cellular senescence mainly by TMT-labeled proteomics and verified the experiment. To find potential molecular targets underlying RIHD and provide new diagnostic and therapeutic methods.

\section{Methods}

\section{Establishment of a mouse RIHD model}

The establishment of a mouse RIHD model and radiation parameters were performed according to a previous study[24]. The whole hearts of 10 experimental C57BL/ 6 male mice (Shanghai Institute of Biochemistry and Cell Biology) aged 9 weeks were irradiated with $16 \mathrm{~Gy} / 1 \mathrm{~F}$ and killed at 1 month (5 mice), and 5 months ( 5 mice) after irradiation. At 5 months, the mice in the control group ( 13 mice) were sacrificed and heart tissues were harvested for the further study. All the mice were intraperitoneally injected with pentobarbital sodium before they were sacrificed. The mice were bred and housed with adequate water and food. The experiment was approved by the Animal Ethics Committee of Second Affiliated Hospital of Nanchang University.

\section{H9C2 Cell line culture}

The $\mathrm{H} 9 \mathrm{C} 2$ cell line was cultured at $37^{\circ} \mathrm{C}$ with $5 \% \mathrm{CO} 2$ in high glucose DMEM (Gibco, USA) with $10 \% \mathrm{FBS}$ (Gibco, USA), $100 \mathrm{U} / \mathrm{ml}$ penicillin, and $50 \mu \mathrm{gg} / \mathrm{ml}$ streptomycin (Solarbio, China) in $10 \mathrm{~cm}$ culture dishes (Nest, China). Then,the cell lines were divided into an irradiation group (Varian Clinic 23EX,USA) and a 
control group with $0 \mathrm{~Gy}$. The irradiated dishes were covered with a $1 \mathrm{~cm}$ bolus and received a single dose of $10 \mathrm{~Gy}$ at a dose rate of $600 \mathrm{cGy} / \mathrm{min}$, and $S S D=100 \mathrm{~cm}$. Cells were harvested after $12 \mathrm{~h}, 24 \mathrm{~h}$ and 48 hours of irradiation. Additionally, control cells harvested after $48 \mathrm{~h}$.

\section{Plasmid constructs and transfection}

The mutant plasmids of Atp5f1c K55Q-6his, Atp5f1c K55R-6his, and Atp5f1c-6his were constructed by Shanghai Jikai Gene Chemical Technology Co., Ltd. (Shanghai, China), and cDNA fragments were cloned into GV417 eukaryotic expression vectors. The resulting PCR product was digested with Nhel/BamHI. Successful mutation was confirmed by DNA sequencing. The plasmid encoding the Atp $5 \mathrm{f} 1 \mathrm{c}$ mutant $\mathrm{K} 55 \mathrm{Q}$ was generated using the following primers: $\mathrm{K} 55 \mathrm{Q}$, forward,5'GAACCGTCAGATCCGCTAGCCGCCACCATGTTCTCGCGGGCGAGCGTTG3',reverse:5'GGAGGGAGAGGGGCGGATCTTAATGATGATGATGATGATGTG3'. The plasmid encoding the mutant K55R was generated using the following primers:K55Rforward:5'GAACCGTCAGATCCGCTAGCCGCCACCATGTTCTCGCGGGCGAGCGTTG3',reverse:5'GGAGGGAGAGGGGCGGATCTTAATGATGATGATGA7 The plasmid encoding the Atp5f1c mutant control was generated using the following primers: Control, forward:5'GAACCGTCAGATCC GCTAGCCGCCACCATGTTCTCGCGGGCGAGCGTTG'.Control,reverse:5'GGAGGGAGAGGGGCGGATCTTAATGATGATGATGATGATGTG3'.

The overexpression plasmids of HA-Sirt3 (NM), HA-SIRT4 and HA-Sirt5 were constructed by Shanghai Jikai Gene Chemical Technology Co., Ltd. (Shanghai, China), and cDNAs fragment were cloned into GV366 eukaryotic expression vectors. The resulting PCR product was digested with BamHI/Xhol. Successful mutation was confirmed by DNA sequencing. The plasmid encoding Sirt3 overexpression was generated using the following primers: Sirt3, forward: 5'ACGGGCCCTCTAGACTCGAGCGCCACCATGGTGGGGGCTGGCATCAG3',reverse:5'TCATAAGGGTACATGGATCCTCCGTCCTGTCCATCCAGCTTTC3'. The plasmid encoding Sirt4 overexpression was generated using the following primers:Sirt4,forward:5'ACGGGCCCTCTAGACTCGAGCGCCACCATGAGGGGGTTGATTTTCAGG3',reverse:5'TCATAAGGGTACATGGATCCCTGTGGGTCTATTAAGG( 3'.The plasmid encoding Sirt5 overexpression was generated using the following primers: Sirt5, forward:

5'ACGGGCCCTCTAGACTCGAGCGCCACCATGCGACCGCTCCCGGTCGC',reverse:5'TCATAAGGGTACATGGATCCAGAAATCCTTTCAGTTTCATG3'.Candidate plasmids were transiently transfected into cells using Lipo3000 (Invitrogen, Waltham, Massachusetts, USA) according to the manufacturer's instructions. After transfection, the cells were harvested after $48 \mathrm{~h}$ and subjected to further analysis.

\section{qPCR}

Total RNA of the heart apex was extracted by TRIzol Invitrogen (Solarbio,China) following manufacturer's instructions and then reverse transcribed into cDNA using the PrimeScript ${ }^{\mathrm{TM}}$ RT reagent Kit with gDNA Eraser (TaKaRa, Japan). PCR amplifications were performed on TB Green Premix Ex Taq ${ }^{\mathrm{TM}}$ (TaKaRa, Japan) following manufacture's instruction. Actin served as control. The primer sequences were listed in Table S1.

\section{Western blotting}

Control and irradiated mouse heart apex tissue or $\mathrm{H} 9 \mathrm{C} 2$ cell protein extraction, protein concentration and Western blotting process were as previously described[14] The primary antibodies used in this study were anti-acetyllysine rabbit pAb (1:1000; Jingjie PTM BioLabs, Inc.China), anti-Atp5f1 monoclonal antibody (1:1000; Proteintech, Wuhan, China), Anti-p21 monoclonal antibody (1:1000; Boster, Wuhan, China), HA-tagged monoclonal antibody (1:20000; Proteintech, Wuhan, China), 6*His-tagged monoclonal antibody (1:10000; Proteintech, Wuhan, China), GAPDH monoclonal antibody (1:20000; Proteintech, Wuhan, China), and Anti-a-tubulin monoclonal antibody (1:1000; Boster, Wuhan, China). Anti-rabbit (1:10000) and anti-mouse intech (1:10000) antibodies were purchased from Proteintech (Wuhan, China).

\section{Co-IP}

Briefly, samples were extracted from control and irradiated $\mathrm{H} 9 \mathrm{C} 2 \mathrm{cells}$ (10 cm dish per sample) using NP-40 Beyotime, China). Co-IP experiments were performed using SureBeads ${ }^{\text {TM }}$ Protein A/G Magnetic Beads (BioRad SureBeads ${ }^{\text {TM }}$ Protein, CA, USA). Briefly, $30 \mu$ l of SureBeads protein A and G was magnetized and washed with $0.1 \%$ PBS/Tween 20 (PBST), and then incubated with $2 \mu \mathrm{g}$ of anti-acetyllysine rabbit pAb (Jingjie PTM BioLabs Inc, Hangzhou, China) or IgG rabbit antibody (Abcam, USA) at RT for $30 \mathrm{~min}$. Afterward, the incubated beads were washed 5 times with $0.1 \%$ PBST, and then the washed beads were incubated with $100 \mu \mathrm{g}$ of extracted protein overnight at $4{ }^{\circ} \mathrm{C}$. The proteins were electrophoresed in SDS-PAGE gels and immunoblotted on a PVDF membrane (Millipore, USA). Membranes were incubated with Atp5f1c rabbit antibodies (1:1000, Proteintech, Wuhan, China) and then detected by peroxidase conjugated secondary antibody (1:20000, Abcam, USA) with ECL Blotting Substrates (China). Membranes were visualized by chemiluminescence (Bio-Rad, USA) and quantitated by ImageJ 14.9 software.

\section{Proteomics of lysine acetylation}

The process of quantitative proteomics through TMT was performed by Jingjie PTM BioLab (Hangzhou) Co. Ltd. The sham-irradiated and 5-month- irradiated heart apexes were ground in liquid nitrogen and lysed in buffer ( $3 \mu \mathrm{M}$ TSA, $8 \mathrm{M}$ urea, $1 \%$ protease inhibitor). The remaining precipitate was removed by centrifugation at $12,000 \mathrm{~g}$ for $10 \mathrm{~min}$ at $4{ }^{\circ} \mathrm{C}$. The subsequent procedures (trypsin digestion, TMT labeling peptides, HPLC fractionation and LC-MS/MS analysis) were performed as previously described [25]. The resulting MS/MS data were analyzed using the MaxQuant search engine (v.1.5.2.8) at Jingjie PTM BioLab (Hangzhou) Co. Ltd. The search method of the database is displayed in the supplementary material (Data S1).

\section{Bioinformatics analysis}

Functional classification and subcellular localization analysis.

A GO annotation proteome was derived from the UniProt-GOA database (ebi.ac.uk/GOA/). The Wolfpsort Database was used to predict the subcellular localization of the proteins(https://www.genscript.com/psort/wolf_psort.html). 
GO annotations can be divided into three categories: biological process (BP), cellular component (CC) and molecular function (MF). A two-tailed Fisher's exact test was used to assess the enrichment of the differentially expressed proteins (DEPs) against all identified proteins. Kyoto Encyclopedia of Genes and Genomes (KEGG) enrichment analysis was performed at https://www.genome.jp/kegg/.

\section{Protein-protein Interaction Network}

All differentially expressed modified protein database accessions or sequences were searched against the STRING database version 11.0 (https://www.string$\mathrm{db}$.org/)for protein-protein interactions. Only interactions between the proteins belonging to the searched dataset were selected, thereby excluding external candidates. STRING defines a metric called the "confidence score" to define interaction confidence; we fetched all interactions that had a confidence score $>0.7$ (high confidence). Interaction networks from STRING were visualized in Cytoscape 3.7.2. Additionally, cytoHubba was used to study essential nodes in the network with 11 methods (DMNC and degree exhibits a satisfactory comparative performance), completed to explore the hub genes [26].

\section{Telomere length measurement}

The examination of relative average telomere length by the q-PCR-based telomere assay described previously[27]. The $\mathrm{Ct}$ values of telomeres ( $\mathrm{T}$ ) and the single copy gene $36 \mathrm{~b} 4(\mathrm{~S})$ were used as the reference genes and were determined by q-PCR. The ratio of telomere (T) repetitive copy number to single copy internal reference gene $(\mathrm{S})$ can be used to assess the relative length of telomeres, while the $\mathrm{T} / \mathrm{S}$ ratio is proportional to telomere length. The calculation formula of $\mathrm{T} / \mathrm{S}$ is as follows: $\mathrm{T} / \mathrm{S}=[2 \mathrm{CT}$ (telomeres) $/ 2 \mathrm{CT}$ (single copy gene) $]=2-\Delta \mathrm{CT}$. Telomere- $\mathrm{F}$ primer (GGTTTTTGAGGGTGAGGGTGAGGGTGAGGGTGAGGGT), Telomere-R primer (TCCCGACTATCCCTATCCCTATCCCTATCCCTATCCCTA). Mouse 36B4-F primer (ACTGGTCTAGGACCCGAGAAG), and mouse 36B4-R primer (TCAATGGTGCCTCTGGAGATT). Telomere PCR was performed at $95^{\circ} \mathrm{C}$ for 10 min, followed by amplification rounds consisting of 40 cycles at $95^{\circ} \mathrm{C}$ for $15 \mathrm{~s}, 60^{\circ} \mathrm{C}$ for $1 \mathrm{~min}$ and $72{ }^{\circ} \mathrm{C}$ for $30 \mathrm{~s}$. The telomere repeat copy number to single gene copy number (T/S) ratio was determined using a Bio-Rad connect in a 96-well format. All samples for both tBopy gene reactions were performed in triplicate.

\section{ATP synthase activity assay}

Mitochondria were isolated using a Cell Mitochondria Isolation Kit囚Beyotime $\mathbb{C} 3601$, China . ATP synthase activity was measured with an ATP synthase enzyme activity microplate assay kit (Abcam, ab109714, US). Briefly, samples of transfected H9C2 cells were collected. ATP synthase from these samples was immunocaptured within the wells, and its enzyme activity was measured by determining the production of ADP, which is coupled with oxidation of nicotinamide adenine dinucleotide hydrogen (NADH) to nicotinamide adenine dinucleotide $\left(\mathrm{NAD}^{+}\right)$and monitored as a decrease in absorbance at 340 nm in accordance with the manufacturer's protocol.

\section{Senescence-associated-galactosidase activity assay}

Transfected cells were isolated and cultured as described above. The cells were incubated with recombinant adiponectin or $25 \mathrm{mmol} / \mathrm{l}$ glucose for $72 \mathrm{~h}$. Cellular senescence was detected using a Senescent Cells Staining Kit (Beyotime, China). Blue-stained cells and total cells were counted, and the percentage of galactosidase-positive cells was calculated.

\section{Cardiac Echocardiogram}

Transthoracic echocardiography was performed using the Vevo 2100 ultrasound system (VisualSonics, Toronto, Canada) according to a previous study[14]. Two-dimensional guided M-mode echoes were obtained at the level of the largest left ventricle (LV). The left ventricular posterior wall at the end of the diastole was measured from the M-mode image. LV ejection fraction (EF) was calculated from the measured ventricle dimensions.

\section{Statistical analysis}

All data are presented as the mean \pm SD of at least three repeats. Statistical differences between multiple comparisons were determined using one-way analysis of variance (ANOVA) with LSD post hoc test and differences between two group comparisons were determined using Student's t-test. All data were analyzed by SPSS version 20 (IBM Corp.). P<0.05 was considered to indicate a statistically significant difference.

\section{Results}

\section{lonizing radiation induces heart and cardiomyocyte senescence}

Ionizing radiation can elicit cellular senescence including cardiomyocyte, fibroblasts, and epithelial cells [28]. Cardiac senescence always exhibits ventricular remodeling, telomere attrition, proinflammatory and profibrotic molecule secretion and activation of the p53, P16 or P21 signaling pathways[29]. A previous study showed that p21-/- mice are prone to more severe RIHD after irradiation than wild-type mice. In this study, signs of radiation-induced mouse cardiac senescence manifested after 5 months. Then, we systematically investigated whether radiation induced cardiac senescence phenotypes. Echocardiography results indicated enlarged cardiac chambers, thinner ventricular walls and decreased left ventricular ejection fraction (Fig. 1A-B). The SASP mRNA level in heart tissue was detected by q-PCR analysis. The results indicated that expression of the fibrosis-related factors MMP9, Trimp1, Col1a1, Col3a1, CTGF, and aSMA and the inflammatory factors IL-1, IL-6, CCL-2, and TNF-a dramatically increased after 5 months of radiation (Fig. 1C-D). Meanwhile, compared with the hearts of the control group, the hearts of the irradiated group exhibited shortened lengths of telomeres (Fig.1E). Aging-related protein P21 also increased with time after radiation in $\mathrm{H} 9 \mathrm{C} 2$ cells (Fig. 1F). 
In a previous study, irradiated heart tissue exhibited significant dysregulation of mitochondrial damage and metabolites, which could be characterized by inner mitochondrial membrane damage and decreased ATP synthesis[24]. Posttranslational protein acetylated modifications are involved in the regulation of metabolism[30].To investigate whether ionizing radiation could drive lysine acetylation changes, we detected the acetylation level of irradiated heart tissue and $\mathrm{H} 9 \mathrm{C} 2$ cells, and the results indicated that high-energy radiation could alter the acetylation status (Fig. 2A-B).

For further analysis of acetylated alterations of irradiated heart proteins, proteomics of lysine acetylation was conducted (Fig. 2C). A total of 721 proteins with 2138 acetylation sites were discovered in the acetylation modification proteome data, of which 666 proteins with 1985 acetylation sites were quantified (Table S1). Sixty-one proteins with 80 acetylation sites were exhibited upregulated, and 9 proteins with 10 acetylation sites were downregulated (Fig. S1A-B).

In accordance with high-throughput acetyl-proteomic analysis of classification and enrichment analysis. Nearly half of the upregulated proteins and acetylation sites were located in mitochondria (Fig S1C). Clusters of Orthologous Groups (COG) analysis was performed and the results revealed that DEPs in acetylation sites mainly clustered in energy production and conversion, lipid transport and metabolism and amino acid transport and metabolism (Fig. 2D). In addition, the GO functional enrichment analysis elucidated that most DEPs of modification sites were enriched in single-organism process, cellular process and metabolic process in the BP category (Fig. 2E). Within the cellular component category, large numbers of DEPs were categorized into mitochondrial categories (Fig. S1D). Metabolic enzymes activity and binding were also enriched in the main MF category (Fig S1E). According to the KEGG database, differentially expressed acetylated proteins were clearly enriched in different metabolic pathways such as butanoate, beta-alanine, amino acid and fatty acid metabolism (Fig. 2F).

Sixty-nine proteins were filtered into the DEP PPI network complex using STRING, and the resultant PPI network contained 52 nodes and 129 edges (Fig. 2 G). The top 10 hub genes selected using the DMNC and degree methods (score $\geq 5,000$ ) and node degree (score $\geq 10$ ) in the cytoHubba plug-in included Atp5c1(Atp5f1c) and HS P901b (Fig. 2H-I).

\section{Hyperacetylated Atp5f1c K55 site induces metabolic dysfunction and cardiomyocyte senescence}

In the above acetyl-proteomics results, DEPs of acetylation modification related to fatty acid metabolism and energy metabolism were obviously overexpressed and were extensively distributed in the mitochondria and mitochondrial membrane. Furthermore, radiation induced cardiac damage and ATP depletion. Therefore, we narrowed our research to the subset of key enzymes involved in metabolism. The mitochondrial membrane ATP synthase subunit was hyperacetylated after radiation in accordance with acetyl proteomics including Atp5f1c, Atp5h and Atp5me (Atp5k), which produced ATP from ADP in the presence of a proton gradient across the membrane that is generated by electron transport complexes of the respiratory chain. Atp5f1c was the most hyperacetylated protein in the ATP synthase subunit (Table 1). The Lys-55 site of Atp $5 \mathrm{f} 1 \mathrm{c}$ was fairly conserved among mammalian species validated by ClustalX software, indicating that it has high fidelity (Fig. $3 \mathrm{~A}$ ). We confirmed alterations in Atp5f1c protein acetylation levels in radiated heart tissue compared to the control by coimmunoprecipitation (Fig. 3B).

Table 1: Modified K sites of ATP synthase subunit by lysine acetylation Proteomic

\begin{tabular}{|lllllll|}
\hline Protein accession & Position & Ratio & Regulated Type & P value & Amino acid & Gene name \\
\hline Q91VR2 & 55 & 1.346 & Up & 0.00080 & $\mathrm{~K}$ & Atp5f1c \\
\hline Q9DCX2 & 95 & 1.202 & Up & 0.048 & $\mathrm{~K}$ & Atp5h \\
\hline Q06185 & 34 & 1.308 & Up & 0.025 & $\mathrm{~K}$ & Atp5me \\
\hline
\end{tabular}

Since Atp $5 \mathrm{f} 1 \mathrm{c}$ is an enzyme central to metabolism, radiation could cause hyperacetylation of cardiac Atp5f1c and Atp5f1c Lys-55 has fidelity in various mammals, we further investigated whether the alterations in Atp $5 \mathrm{f} 1 \mathrm{c}$ Lys-55 acetylation mediated cardiac senescence and energy metabolism caused by radiation. Subsequently, we constructed plasmids purified His proteins containing the mutant site of Atp5f1c Lys-55 to GIn (mimic acetyl-modification, K55Q) and $\mathrm{Arg}$ (mimic deacetyl-modification, K55R) in the $\mathrm{H} 9 \mathrm{C} 2$ cell line. The expression of His tag protein showed that the plasmid was successfully transfected into $\mathrm{H} 9 \mathrm{C} 2$ cells (Fig. $3 \mathrm{C}$ ). To investigate the effect of $\mathrm{K} 55$ acetylation of Atp5f1c on senescence and energy metabolism, plasmids with $\mathrm{K} 55 \mathrm{Q}$, K55R and empty vector were transfected into $\mathrm{H} 9 \mathrm{C} 2$ cells. The hyperacetylated Atp5f1c Lys-55 site exhibited decreased ATP synthesis enzymatic activity, and decreased ATP synthesis was also simultaneously detected (Fig. 3D-E). Concurrently, senescent H9C2 cells had increased (Fig. 3F-G) and P21 protein was clearly overexpressed in the K55Q (mimic acetyl-modification) group (Fig. $3 \mathrm{H}-\mathrm{I}$ ).

\section{Sirt4 and Sitr5 mediate Atp5f1c K55-Ac deacetylation}

The above experiments demonstrated that hyperacetylation of the Atp $5 f 1 \mathrm{c}$ Lys- 55 site led to myocardial cell senescence by affecting mitochondrial ATP synthesis. Acetylation of nonhistone proteins is regulated by the classical HDAC family and NAD+-dependent Sirt family. Then, we investigate the effect of the SIRT inhibitor nicotinamide (NAM) and HDAC inhibitor trichostatin A (TSA) on ATP5f1c acetylation. Since NAM treatment clearly increased Atp5f1c acetylation (Fig. 4A), we identified that nicotinamide adenine dinucleotide (NAD+)-dependent Sirts are involved in Atp5f1c acetylation. Considering that Atp5f1c was localized in the mitochondria, the major mitochondrial deacetylases Sirt3, Sirt4 and Sirt5 were selected for further investigation. H9C2 cells were transfected with empty vector, Sirt3 overexpression plasmid, Sirt4 overexpression plasmid and Sirt5 overexpression plasmid with HA-tag. The presence of the HA-tag was used to demonstrate expression of the above Sirts plasmid (Fig. 4B). The decreased level of Atp5f1c-Ac was validated by Co-IP, which demonstrated that Sirt4 and Sitr5 mediated Atp5f1c protein acetylation levels (Fig. 4C). 


\section{Discussion}

Cellular senescence is a permanent state of cell cycle arrest that promotes tissue remodeling and often takes place in different physiological and pathological processes [31, 32].DNA damage induced double-strand breaks (DSBs) have gradually been recognized as a significant factor in senescence [33]. lonizing radiation leading to cellular senescence was verified by decreased cardiac function, shortened telomeres, and aggravated expression of proaging markers $\mathrm{P} 21$

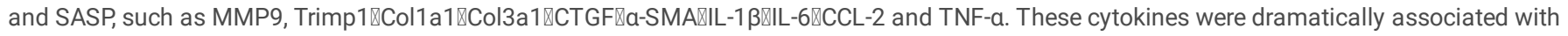
inflammation and fibrosis. Philipp $\mathrm{J}$ et al. indicated that irradiated endothelial cells may disadvantageously affect surrounding normal cells via SASP, which correlated with radiation-induced heart damage [34]. In addition to the heart, several studies have reported that radiation induces senescence and even increases the risk of secondary neoplasms in other normal organs[35, 36]. RIHD arises from cell apoptosis and inflammatory and fibrotic processes, which can lead to the thickening of blood vessels and fibrotic scar tissue in the heart[37]. Several studies have linked altered lysine acetylation with the development of radiation induced damage[38]. In this study, acetylated protein levels were extensively elevated compared to sham-irradiated heart tissue. The same acetylated alterations were verified in $\mathrm{H} 9 \mathrm{C} 2$ cells. Subsequent subcellular localization and functional enrichment analysis from acetylproteomics indicated that DEPs of different acetylated sites were enriched in mitochondrial and energy metabolism. Interestingly, we discovered that irradiated heart tissue exhibited significant dysregulation of mitochondrial damage and metabolites in a previous study. In addition to the myocardium, radiation-induced skeletal muscle mitochondrial damage also altered proteins involved in energy metabolism-related processes[39]. Lysine acetylation-mediated metabolic regulation was involved in radiation-induced senescence.

Atp5f1c is a component of an ATP synthase complex located in the mitochondrial inner membrane that produces ATP from ADP in the presence of a proton gradient across the membrane that is generated by electron transport complexes of the respiratory chain[40]. The current study indicated that bedaquiline, which is a drug that can silence Atpf1c expression in vitro by targeting the gamma subunit of the ATP synthase, was approved by the FDA to inhibit mitochondrial ATP production[41]. Moreover, mitochondrial ATP synthase may be a potential drug target for aging in the investigation of Alzheimer's disease through activation of the typical longevity AMPK/mTOR pathway[42]. Atp5f1c is an important enzyme in metabolic pathways. In this study, ionizing radiation significantly increased Atp5flc K55 acetylation levels and the overexpression of Atp5f1c K55Ac induced metabolic disorder.

Acetylation level of mitochondrial metabolic enzymes acetylated or deacetylated by NAD ${ }^{+}$dependent deacetylases sirtuins (Sirts) [43]. Sirts were NAD ${ }^{+}$ dependent deacetylases and ADP-ribosyltransferases related to energy metabolism and senescence.[44, 45] The mitochondrial sirtuins Sirt3,Sirt4 and Sirt5 are mainly located on and involved in metabolic reactions and antioxidant properties[46]. The ratio between NAD ${ }^{+}$and NADH has gradually been investigated in cellular and mitochondrial metabolism, which had close relationship with glycolysis and the tricarboxylic (TCA) cycle[47]. The level of NAD+ in mammalian cells and tissues declines with aging [48]. NAD -dependent enzyme Sirt loss of function has been linked to aging-related diseases such as cancer, insulin resistance, heart disease, fibrosis, and neurodegeneration[49].

In the human myocardium of heart failure, Zhang XK et al. observed that a decrease in of miR-195 and mitochondrial deacetylase Sirt3, which enhanced ATP synthase acetylation and influenced cardiac energy metabolism[50].Label-free quantitative proteomics of mouse liver mitochondria indicated the absence of Sirt3 regulated acetylation of multiple metabolism-related proteins[51]. Loss of mitochondrial Sirt function, especially Sirt3, has been linked to several agerelated pathologies, including cancer, insulin resistance, heart disease, fibrosis, and neurodegeneration. Generally speaking, SIRT3 is regarded as the major deacetylase in mitochondria[52].However, in our study, overexpression of both Sirt4 and Sirt5 decreased the acetylation level of Atp5flc. Protein activity is regulated through deacetylation of lysine residues.Sirt4 also has an important role in insulin secretion, fatty acid oxidation, amino acid metabolism, ATP homeostasis, cardiovascular diseases and so on [44]. Sirt4 deacetylated malonyl CoA decarboxylase (MCD) and decreased enzyme activity, limiting fatty acid oxidation under adequate nutrition conditions[53]. Liang Guo et al. suggested that Sirt4 dramatically deacetylated the MTPa K350-Ac, K383-Ac,and K406-Ac sites and that MTPa acetylation played an important role in lipid catabolism in nonalcoholic fatty liver disease[54].As with Sirt4, Sirt5 also had an important role in metabolic adaptations. Sirt5 appeared to work in the regulation of heart function. Sirt5 KO mice developed hypertrophic cardiomyopathy and showed reduced cardiac function during aging. Although sirt3, sirt4, sirt5 are mainly localized in mitochondrial matrix[47], inhibiting Sirt4 increased fat oxidative capacity and mitochondrial function in liver and muscle cells[55] while the liver of Sirt3 $\%$ mice decreased $\beta$-oxidation of fatty acids [56]. Even the same type of deacetylase exerts different functions.

In this study, we revealed that ionizing radiation could indeed induce cardiomyocyte senescence and regulate the acetylation level of Atp5f1c, a key enzyme of energy metabolism, providing novel insight into radiation-induced senescence mediated by metabolic regulation. This is the first study on the role of Atp5f1c K55 acetylation modification in radiation-induced heart disease by affecting cardiomyocyte ATP production and cell senescence, and found that Sirt4 and Sirt5 can mediate Atp5f1c deacetylation. This provides a sufficient theoretical basis for elucidating the pathogenic mechanism of radiation heart disease and finding potential therapeutic targets.

\section{Conclusion}

lonizing radiation induced cardiomyocyte senescence and energy metabolism disorder. Atp5f1c K55-ac, which is modulated by Sirt4 or Sirt5, might be a potential molecular target underlying RIHD.

\section{Abbreviations}

IR: Ionizing radiation; RIHD: Radiation-induced heart damage; SASP: Senescence-associated secretory phenotype; TBI: Total-body irradiated; KATs: Lysine acetyltransferases; KDACs: Lysine deacetylases; NADH: Nicotinamide adenine dinucleotide hydrogen; NAD+: Nicotinamide adenine dinucleotide; BP: Biological process, CC: Cellular component; MF: Molecular function; DEPs: Differentially expressed proteins; KEGG: Kyoto Encyclopedia of Genes and Genomes; LV: Left ventricle; EF: Ejection fraction; COG: Clusters of Orthologous Groups; NAM: Nicotinamide; TSA: Trichostatin A; MCD: Malonyl CoA decarboxylase. 


\section{Declarations}

\section{- Ethical Approval}

The experiment was approved by the Animal Ethics Committee of Second Affiliated Hospital of Nanchang University.

\section{- Consent for publication}

Not applicable.

\section{- Availability of supporting data}

The data used to support the finding of this study are available from the corresponding author upon request

\section{- Competing interests}

The authors declare that they have no competing interests.

\section{- Funding}

This study was supported by the National Natural Science Foundation of China [grant numbers 81960571 and 82060577], Science and Technology Innovation Outstanding Young talents training Program of Jiangxi Province [grant number20192BCBL23023]. Postgraduates Special Funds Innovation of Jiangxi Province [grant numbers YC2020-B048].

\section{- Authors' contributions}

Anwen Liu and Zhimin Zeng contributed to the conception and design of the review. Peng Xu and Zhimin Zeng wrote the manuscript. Long Huang and Yanqing Herevised the final manuscript. Peng Xu, Yali Yi and Zhicheng Liu performed experiments. Zhimin Zeng, Yanqing He and Peng Xu performed bioinformatics analysis. Long Huang and Jing Cai gave final approval of the version to be published and participated in data analysis. All authors approved the final version of the manuscript.

\section{- Acknowledgements}

An earlier version of this paper was presented at the 2020 ASTRO Annual Meeting. We thank the Zhejiang key laboratory of radiation oncology for supporting our work of establishing the animal model. We thank Jingjie PTM Biolab Co. Ltd for conducting and analyzing proteomics. Thanks to the reviewers of this manuscript for their valuable suggestions.

\section{References}

1. Moding EJ, Kastan MB, Kirsch DG: Strategies for optimizing the response of cancer and normal tissues to radiation. Nature reviews Drug discovery 2013, 12(7):526-542.

2. Straub JM, New J, Hamilton CD, Lominska C, Shnayder Y, Thomas SM: Radiation-induced fibrosis: mechanisms and implications for therapy. J Cancer Res Clin Oncol 2015, 141(11):1985-1994.

3. Simone CB, 2nd: Thoracic Radiation Normal Tissue Injury. Semin Radiat Onco/ 2017, 27(4):370-377.

4. Mahdavi H: Radiation oncologists' perspectives on reducing radiation-induced heart disease in early breast cancer. Curr Prob/ Cancer 2020, 44(2):100509.

5. van Nimwegen FA, Ntentas G, Darby SC, Schaapveld M, Hauptmann M, Lugtenburg PJ, Janus CPM, Daniels L, van Leeuwen FE, Cutter DJ et al: Risk of heart failure in survivors of Hodgkin lymphoma: effects of cardiac exposure to radiation and anthracyclines. Blood 2017, 129(16):2257-2265.

6. De Ruysscher D, Niedermann G, Burnet NG, Siva S, Lee AWM, Hegi-Johnson F: Radiotherapy toxicity. Nature Reviews Disease Primers 2019, 5(1).

7. Steiner I: Pathology of radiation induced heart disease. Reports of practical oncology and radiotherapy : journal of Greatpoland Cancer Center in Poznan and Polish Society of Radiation Oncology 2020, 25(2):178-181.

8. He S, Sharpless NE: Senescence in Health and Disease. Cell 2017, 169(6):1000-1011.

9. Gude NA, Broughton KM, Firouzi F, Sussman MA: Cardiac ageing: extrinsic and intrinsic factors in cellular renewal and senescence. Nat Rev Cardio/ 2018, 15(9):523-542.

10. Hinkal GW, Gatza CE, Parikh N, Donehower LA: Altered senescence, apoptosis, and DNA damage response in a mutant p53 model of accelerated aging Mech Ageing Dev 2009, 130(4):262-271.

11. Liu X, Shang S, Chu W, Ma L, Jiang C, Ding Y, Wang J, Zhang S, Shao B: Astragaloside IV ameliorates radiation-induced senescence via antioxidative mechanism. J Pharm Pharmacol 2020, 72(8):1110-1118.

12. Costantino S, Paneni F, Cosentino F: Ageing, metabolism and cardiovascular disease. The Journal of Physiology 2016, 594(8):2061-2073. 
13. Guzik TJ, Cosentino F: Epigenetics and Immunometabolism in Diabetes and Aging. Antioxidants \& redox signaling 2018, 29(3):257-274.

14. Zeng ZM, Du HY, Xiong L, Zeng XL, Zhang P, Cai J, Huang L, Liu AW: BRCA1 protects cardiac microvascular endothelial cells against irradiation by regulating p21-mediated cell cycle arrest. Life Sci 2020, 244:117342.

15. Unthank JL, Ortiz M, Trivedi H, Pelus LM, Sampson CH, Sellamuthu R, Fisher A, Chua HL, Plett A, Orschell CM et al: Cardiac and Renal Delayed Effects of Acute Radiation Exposure: Organ Differences in Vasculopathy, Inflammation, Senescence and Oxidative Balance. Radiat Res 2019, 191(5):383-397.

16. Menzies KJ, Zhang H, Katsyuba E, Auwerx J: Protein acetylation in metabolism - metabolites and cofactors. Nat Rev Endocrinol 2016, 12(1):43-60.

17. Narita T, Weinert BT, Choudhary C: Functions and mechanisms of non-histone protein acetylation. 2019, 20(3):156-174.

18. Li P, Ge J, Li H: Lysine acetyltransferases and lysine deacetylases as targets for cardiovascular disease. Nat Rev Cardio/ 2020, 17(2):96-115.

19. Gil J, Ramírez-Torres A, Encarnación-Guevara S: Lysine acetylation and cancer: A proteomics perspective. J Proteomics 2017, 150:297-309.

20. Min Z, Long X, Zhao H, Zhen X, Li R, Li M, Fan Y, Yu Y, Zhao Y, Qiao J: Protein Lysine Acetylation in Ovarian Granulosa Cells Affects Metabolic Homeostasis and Clinical Presentations of Women With Polycystic Ovary Syndrome. Frontiers in cell and developmental biology 2020, 8:567028.

21. Feng X, Zhang L, Xu S, Shen AZ: ATP-citrate lyase (ACLY) in lipid metabolism and atherosclerosis: An updated review. Prog Lipid Res 2020, 77:101006.

22. Tsuda M, Fukushima A, Matsumoto J, Takada S, Kakutani N, Nambu H, Yamanashi K, Furihata T, Yokota T, Okita K et al: Protein acetylation in skeletal muscle mitochondria is involved in impaired fatty acid oxidation and exercise intolerance in heart failure. Journal of cachexia, sarcopenia and muscle 2018 , 9(5):844-859.

23. Barjaktarovic Z, Merl-Pham J, Braga-Tanaka I, Tanaka S, Hauck SM, Saran A, Mancuso M, Atkinson MJ, Tapio S, Azimzadeh O: Hyperacetylation of Cardiac Mitochondrial Proteins Is Associated with Metabolic Impairment and Sirtuin Downregulation after Chronic Total Body Irradiation of ApoE ( $/-$ ) Mice. International journal of molecular sciences 2019, 20(20).

24. Xu P, Yi Y, Luo Y, Liu Z, Xu Y, Cai J, Zeng Z, Liu A: Radiation-induced dysfunction of energy metabolism in the heart results in the fibrosis of cardiac tissues Mol Med Rep 2021, 24(6).

25. Zhang L, Zhao SQ, Zhang J, Sun Y, Xie YL, Liu YB, Ma CC, Jiang BG, Liao XY, Li WF et al: Proteomic Analysis of Vesicle-Producing Pseudomonas aeruginosa PAO1 Exposed to X-Ray Irradiation. Frontiers in microbiology 2020, 11:558233.

26. Chin $\mathrm{CH}$, Chen SH, Wu HH, Ho CW, Ko MT, Lin CY: cytoHubba: identifying hub objects and sub-networks from complex interactome. BMC Syst Bio/ 2014, 8 Suppl 4(Suppl 4):S11.

27. Liu B, Maekawa T, Yoshida K, Ly NH, Inoue K, Hasegawa A, Chatton B, Ogura A, Ishii S: Telomere shortening by transgenerational transmission of TNF-ainduced TERRA via ATF7. Nucleic Acids Res 2019, 47(1):283-298.

28. Chen Z, Cao K, Xia Y, Li Y, Hou Y, Wang L, Li L, Chang L, Li W: Cellular senescence in ionizing radiation (Review). Oncol Rep 2019, 42(3):883-894.

29. Shimizu I, Minamino T: Cellular senescence in cardiac diseases. J Cardiol 2019, 74(4):313-319.

30. Ali I, Conrad RJ, Verdin E, Ott M: Lysine Acetylation Goes Global: From Epigenetics to Metabolism and Therapeutics. Chem Rev 2018, 118(3):1216-1252.

31. Calcinotto A, Kohli J, Zagato E, Pellegrini L, Demaria M, Alimonti A: Cellular Senescence: Aging, Cancer, and Injury. Physiol Rev 2019, 99(2):1047-1078.

32. Hernandez-Segura A, Nehme J, Demaria M: Hallmarks of Cellular Senescence. Trends Cell Biol 2018, 28(6):436-453.

33. Ou HL, Schumacher B: DNA damage responses and p53 in the aging process. 2018, 131(5):488-495.

34. Philipp J, Azimzadeh O: Radiation-Induced Endothelial Inflammation Is Transferred via the Secretome to Recipient Cells in a STAT-Mediated Process. 2017, 16(10):3903-3916.

35. Chandra A, Lagnado AB, Farr JN, Monroe DG, Park S, Hachfeld C, Tchkonia T, Kirkland JL, Khosla S, Passos JF et al: Targeted Reduction of Senescent Cell Burden Alleviates Focal Radiotherapy-Related Bone Loss. J Bone Miner Res 2020, 35(6):1119-1131.

36. Yang X, Ren H, Guo X, Hu C, Fu J: Radiation-induced skin injury: pathogenesis, treatment, and management. Aging (Albany NY) 2020, 12(22):23379-23393.

37. Sarkozy M, Varga Z, Gaspar R, Szucs G, Kovacs MG, Kovacs ZZA, Dux L, Kahan Z, Csont T: Pathomechanisms and therapeutic opportunities in radiationinduced heart disease: from bench to bedside. Clin Res Cardiol 2021, 110(4):507-531.

38. Barjaktarovic Z, Merl-Pham J, Azimzadeh O, Kempf SJ, Raj K, Atkinson MJ, Tapio S: Low-dose radiation differentially regulates protein acetylation and histone deacetylase expression in human coronary artery endothelial cells. Int J Radiat Bio/ 2017, 93(2):156-164.

39. Kim EJ, Lee M, Kim DY, Kim KI, Yi JY: Mechanisms of Energy Metabolism in Skeletal Muscle Mitochondria Following Radiation Exposure. Cells 2019, 8(9). 
40. Srivastava AP, Luo M: High-resolution cryo-EM analysis of the yeast ATP synthase in a lipid membrane. 2018, 360(6389).

41. Fiorillo M, Scatena C, Naccarato AG, Sotgia F, Lisanti MP: Bedaquiline, an FDA-approved drug, inhibits mitochondrial ATP production and metastasis in vivo, by targeting the gamma subunit (ATP5F1C) of the ATP synthase. 2021.

42. Goldberg J, Currais A, Prior M, Fischer W, Chiruta C, Ratliff E, Daugherty D, Dargusch R, Finley K, Esparza-Moltó PB et al: The mitochondrial ATP synthase is a shared drug target for aging and dementia. Aging cell 2018, 17(2).

43. Parodi-Rullán RM, Chapa-Dubocq XR, Javadov S: Acetylation of Mitochondrial Proteins in the Heart: The Role of SIRT3. Front Physio/2018, 9:1094.

44. Han Y, Zhou S, Coetzee S, Chen A: SIRT4 and Its Roles in Energy and Redox Metabolism in Health, Disease and During Exercise. Front Physio/ 2019, $10: 1006$.

45. Singh CK, Chhabra G, Ndiaye MA, Garcia-Peterson LM, Mack NJ, Ahmad N: The Role of Sirtuins in Antioxidant and Redox Signaling. Antioxidants \& redox signaling 2018, 28(8):643-661.

46. Min Z, Gao J, Yu Y: The Roles of Mitochondrial SIRT4 in Cellular Metabolism. Front Endocrinol (Lausanne) 2018, 9:783.

47. van de Ven RAH, Santos D, Haigis MC: Mitochondrial Sirtuins and Molecular Mechanisms of Aging. Trends Mol Med 2017, 23(4):320-331.

48. Covarrubias AJ, Perrone R: NAD(+) metabolism and its roles in cellular processes during ageing. 2021, 22(2):119-141.

49. McDonnell E, Peterson BS, Bomze HM, Hirschey MD: SIRT3 regulates progression and development of diseases of aging. Trends Endocrinol Metab 2015, 26(9):486-492.

50. Zhang X, Ji R, Liao X, Castillero E, Kennel PJ, Brunjes DL, Franz M, Möbius-Winkler S, Drosatos K, George I et al: MicroRNA-195 Regulates Metabolism in Failing Myocardium Via Alterations in Sirtuin 3 Expression and Mitochondrial Protein Acetylation. Circulation 2018, 137(19):2052-2067.

51. Rardin MJ, Newman JC, Held JM, Cusack MP, Sorensen DJ, Li B, Schilling B, Mooney SD, Kahn CR, Verdin E et al: Label-free quantitative proteomics of the lysine acetylome in mitochondria identifies substrates of SIRT3 in metabolic pathways. Proc Natl Acad Sci U S A 2013, 110(16):6601-6606.

52. Onyango P, Celic I, McCaffery JM, Boeke JD, Feinberg AP: SIRT3, a human SIR2 homologue, is an NAD-dependent deacetylase localized to mitochondria. Proc Natl Acad Sci U S A 2002, 99(21):13653-13658.

53. Laurent G, German NJ, Saha AK, de Boer VC, Davies M, Koves TR, Dephoure N, Fischer F, Boanca G, Vaitheesvaran B et al: SIRT4 coordinates the balance between lipid synthesis and catabolism by repressing malonyl CoA decarboxylase. Mol Cell 2013, 50(5):686-698.

54. Guo L, Zhou SR, Wei XB, Liu Y, Chang XX, Liu Y, Ge X, Dou X, Huang HY, Qian SW et al: Acetylation of Mitochondrial Trifunctional Protein a-Subunit Enhances Its Stability To Promote Fatty Acid Oxidation and Is Decreased in Nonalcoholic Fatty Liver Disease. Mol Cel/ Bio/ 2016, 36(20):2553-2567.

55. Nasrin N, Wu X, Fortier E, Feng Y, Bare OC, Chen S, Ren X, Wu Z, Streeper RS, Bordone L: SIRT4 regulates fatty acid oxidation and mitochondrial gene expression in liver and muscle cells. J Biol Chem 2010, 285(42):31995-32002.

56. Hirschey MD, Shimazu T, Goetzman E, Jing E, Schwer B, Lombard DB, Grueter CA, Harris C, Biddinger S, Ilkayeva OR et al: SIRT3 regulates mitochondrial fatty-acid oxidation by reversible enzyme deacetylation. Nature 2010, 464(7285):121-125.

\section{Figures}


A
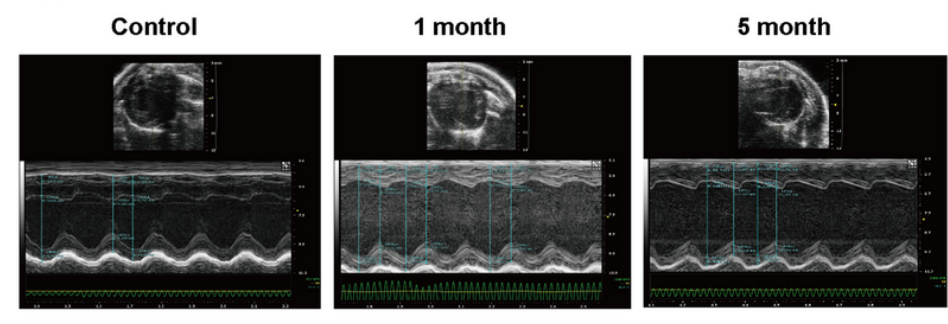

B
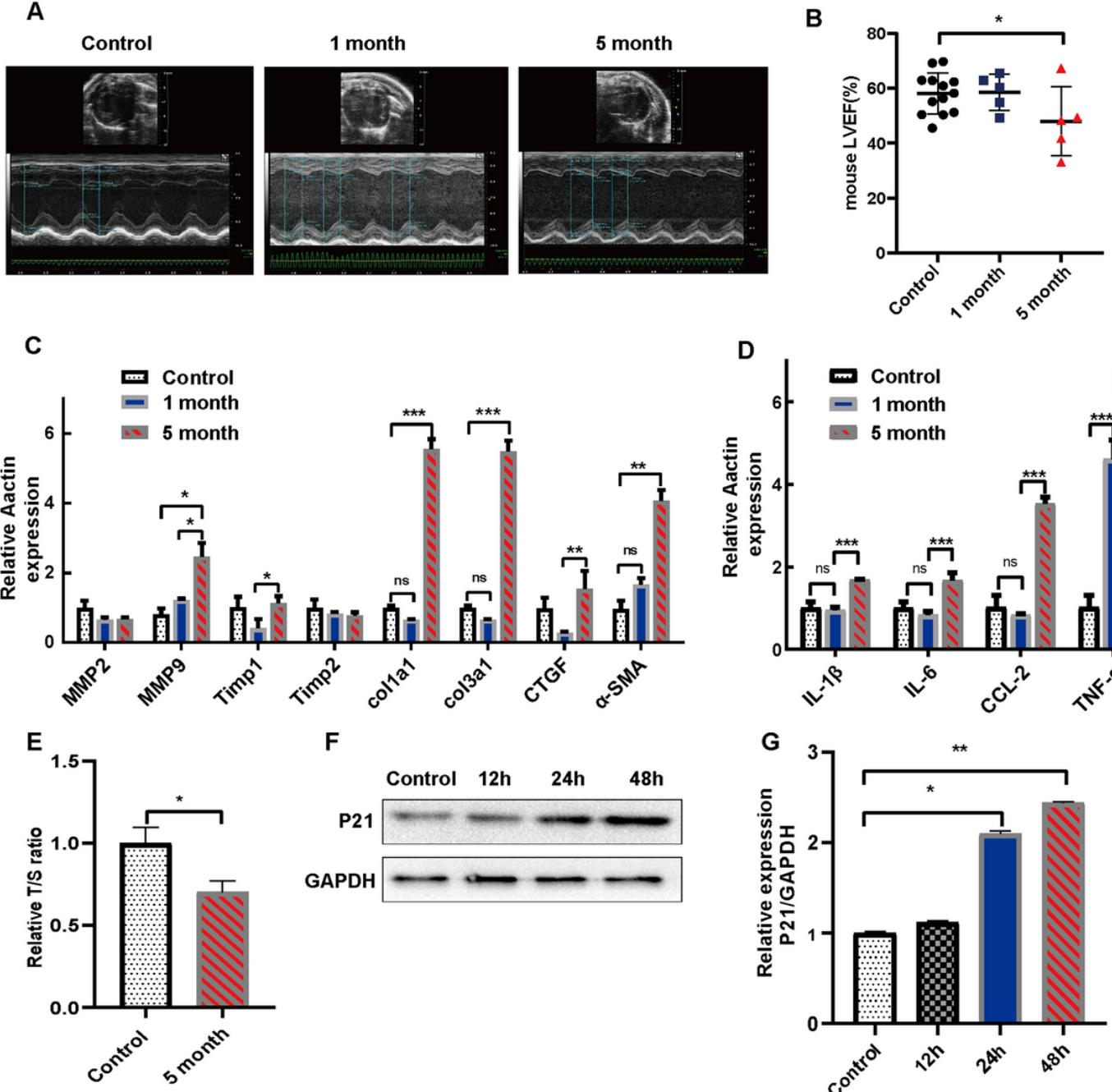

D

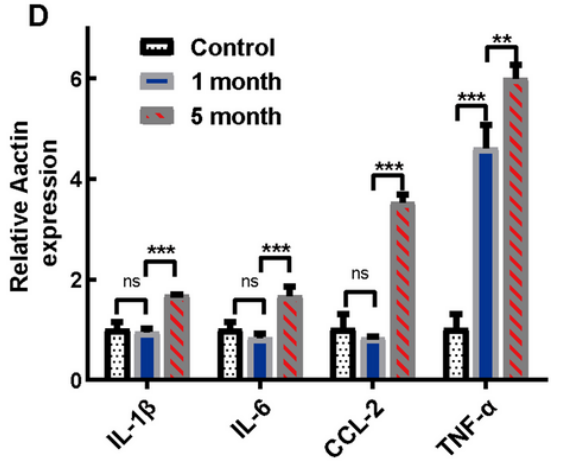

$\mathbf{F}$

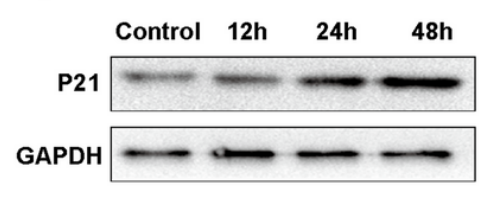

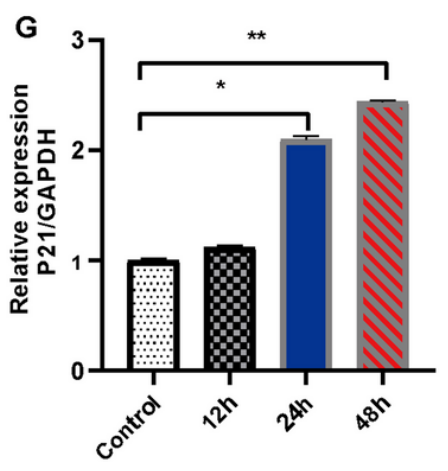

Figure 1

lonizing radiation causes myocardial and heart tissue senescence. (A-B) Echocardiograph images and LVEF of mouse hearts in the control mice, 1 and 5 months after local heart irradiation with a dose of $16 \mathrm{~Gy}$. (C) q-PCR analysis mRNA of fibrosis-associated factors in 1 month, 5 month and control mice (* $p<$ $0.05, * \star p<0.01, \star \star \star p<0.001$, NS, not significant). (D) q-PCR analysis of the mRNA levels of inflammation-associated factors in 1 month, 5 months and control mice ( ${ }^{*} p<0.05, * * p<0.01, * * * p<0.001$, NS, not significant). (E) q-PCR analysis of telomere length in cardiac tissue from 1-month-old, 5-month-old and control mice $(* \mathrm{p}<0.05)$. (F-Q) Western blot and quantification analysis of p21 protein expression after $0,6 \mathrm{~h}, 12 \mathrm{~h}$ and $24 \mathrm{~h}$ irradiation. (* $\mathrm{p}<0.05$, ** $\mathrm{p}<$ $0.01)$. 


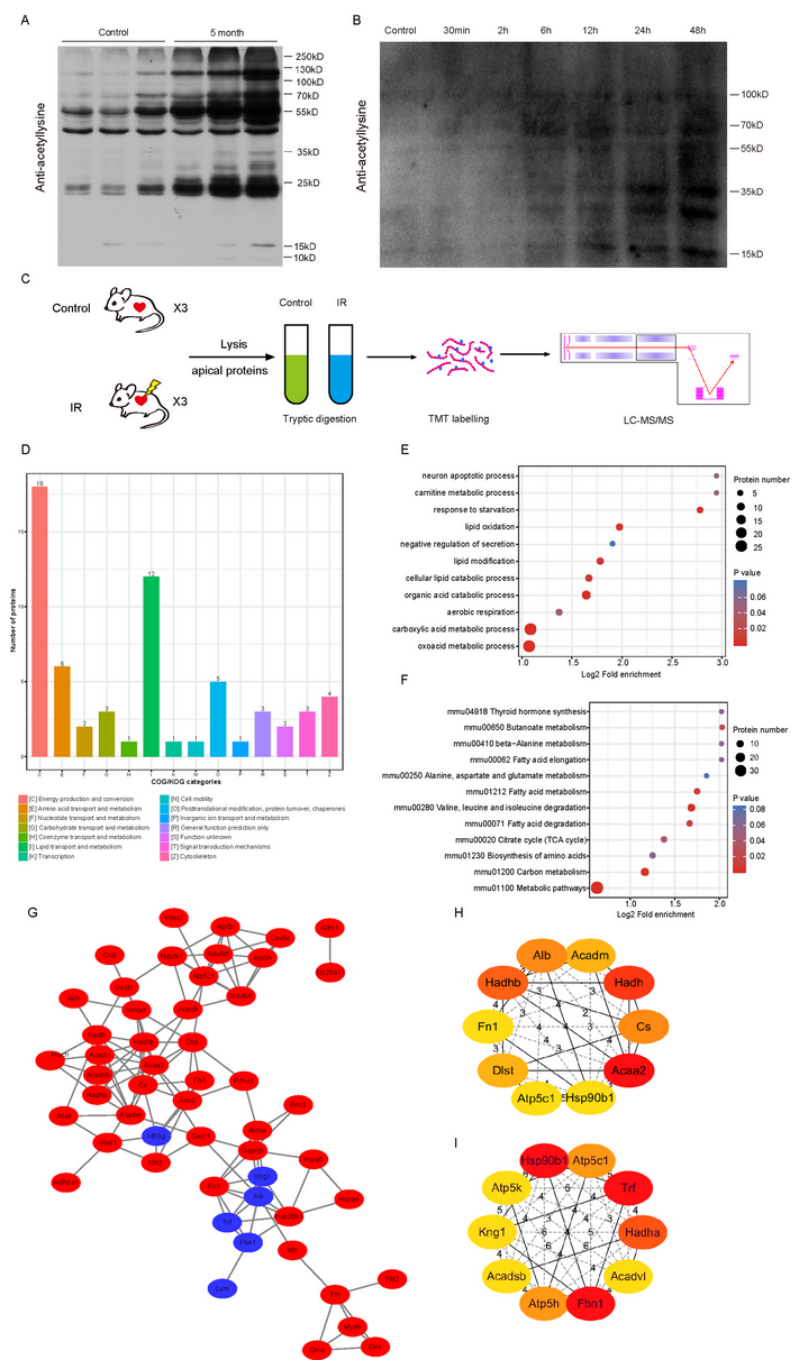

Figure 2

lonizing radiation induces lysine residue hyperacetylation modifies cardiac metabolic enzymes. (A) Radiation induces protein acetylation in mouse hearts after 5 months. (B) Radiation induces protein acetylation in $\mathrm{H9C2}$ cells at different time points. (C) Experimental flow chart of the proteomic analysis. (D) COG/KOG functional classification chart of proteins corresponding to differentially expressed modification sites. (E) GO (Gene Ontology) enrichment bubble plot of proteins corresponding to differentially expressed modification sites in the biological process category. (F) KEGG pathway enrichment bubble plot of proteins corresponding to differentially expressed modification sites. (G) PPI network of the DEPs. Red nodes represent upregulated proteins, and blue nodes represent downregulated proteins. $(\mathbf{H})$ The cytoHubba DMNC and degree methods were used to extract the top 10 hub proteins from the PPI network. (I) cytoHubba of degree methods was used to extract the top 10 hub proteins from the PPI network. 
A

Mus musculus (Mouse)

Rattus norvegicus (Rat)

Homo sapiens (Human)

Pan troglodytes (Chimpanzee)

Macaca fascicularis (Cynomolgus monkey)

Bos taurus (Bovine)
$\mathbf{K}-\mathbf{A c}$

50 M VAAAK Y A AE 60

25 M V A A A K A R A E 35

50 MVA A A K Y A AE 60

50 MVAAAK

M V A A A:K:Y A R A E 60

50 MVAAAK Y Y AE 60

B

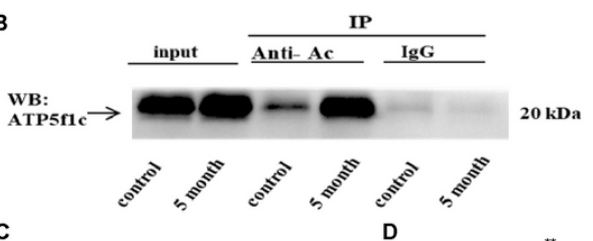

C

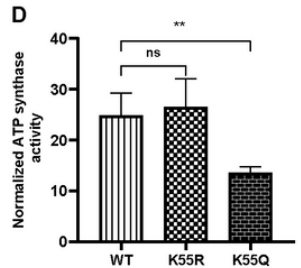

E
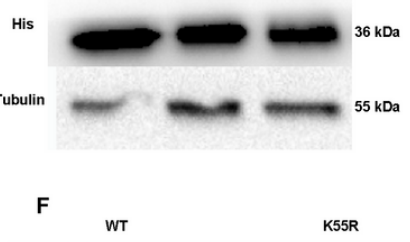

WT

$K 55 R$

K55Q
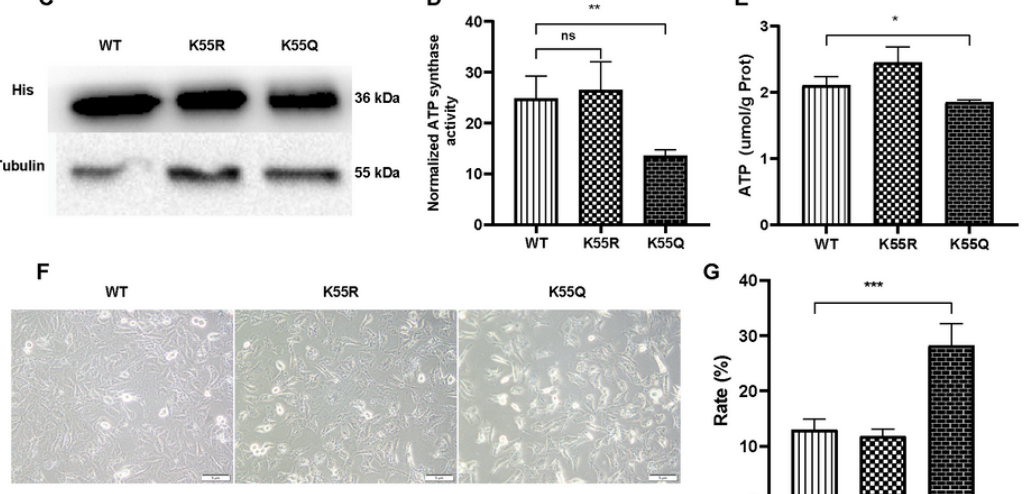

G

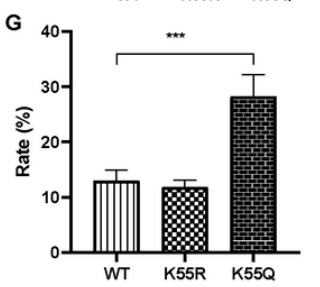

H
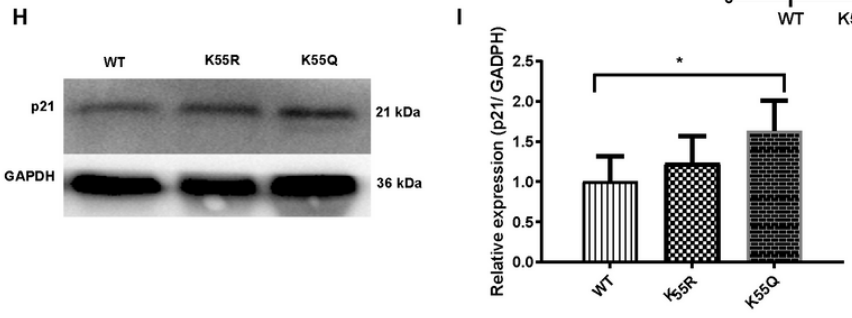

Figure 3

Atp5f1c is hyperacetylated at the 55th lysine site and leads to metabolic disorders and senescence. (A) Sequence alignment of the mouse, rat, human, chimpanzee, monkey and bovine ATP5f1c proteins. (B) lonizing radiation induced ATP5f1c acetylation in heart tissue verified by Co-IP. (C) The expression of anti-His antibody after His-tagged ATP5f1c control, K55R and K55Q plasmids transfection in H9C2 cells. (D-E) ATP synthase activity and ATP production after ATP5f1c control, K55R and K55Q plasmids were transfected into H9C2 cells ( ${ }^{*} p<0.05, * \star p<0.01, * \star * p<0.001$, NS, not significant). (F-G) Senescenceassociated $\beta$-galactosidase (SABG) staining and statistical analysis after ATP5f1c control, K55R and K55Q plasmids transfected in H9C2 cells (* $\mathrm{p}<0.05$, ** $\mathrm{p}$ $<0.01, * * * p<0.001$, NS, not significant). (H-I) Western blotting and statistical analysis of senescence-associated protein P21 in H9C2 cells. Relative protein levels were normalized to tubulin (* $p<0.05$, ** $p<0.01$, *** $p<0.001$, NS, not significant). 
A

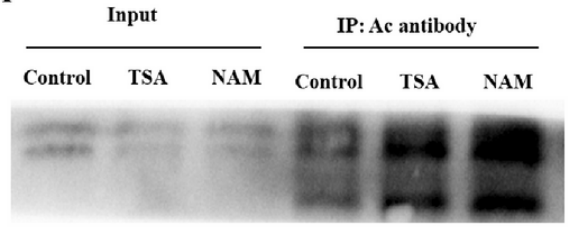

WB :

ATP5f1c

Light chain
B
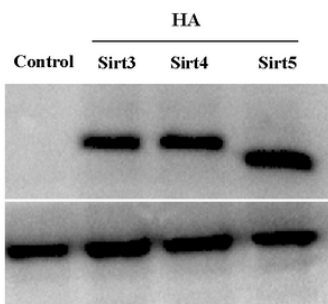

C

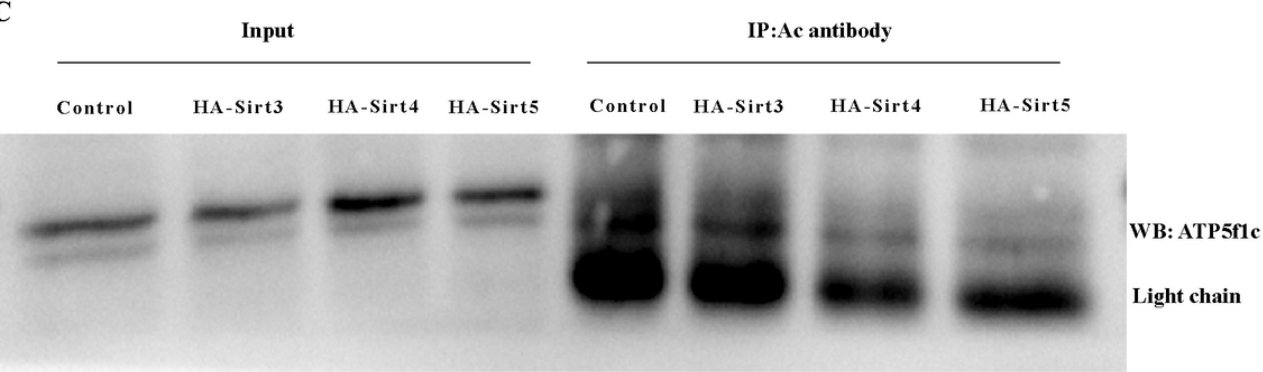

D

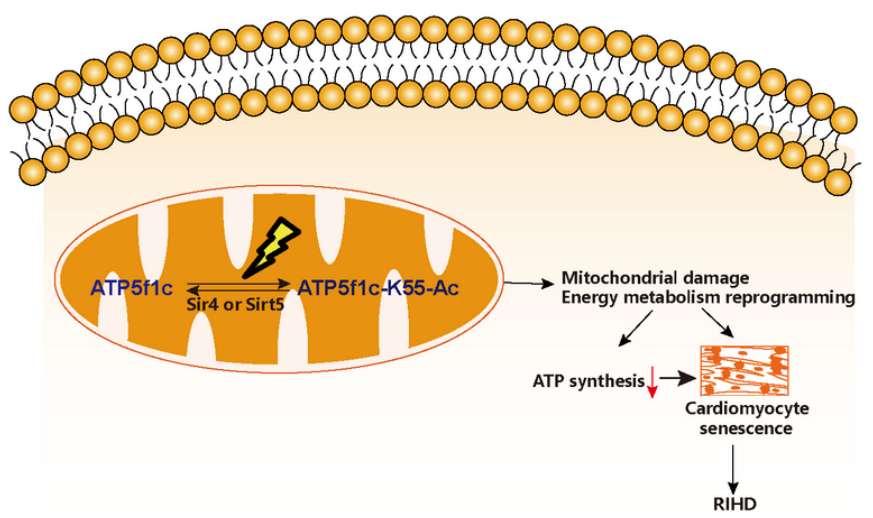

Cardiomyocyte

Figure 4

Sirt4 and Sitr5 mediated Atp5f1c K55- Ac deacetylation (A) Effect of TSA and NAM on ATP5f1c acetylation levels in H9C2 cells. (B) The expression of HA-tag after HA-tagged Sirt3, Sirt4 and Sirt5 plasmids were transfected into H9C2 cells. (C) ATP5f1c acetylation verified by Co-IP after HA-tagged Sirt3, Sirt4 and Sirt5 plasmids were transfected into H9C2 cells. (D) Schematic diagram of the study design.

\section{Supplementary Files}

This is a list of supplementary files associated with this preprint. Click to download.

- SupplementaryMaterial.zip 